\title{
PROPENSITY AND POSSIBILITY
}

(1) In his book Aquinas Anthony Kenny says on page 29/30: "Interest in logical formalisms which obliterate the distinctions between the potential and the actual ('possible worlds') is currently more fashionable than the serious study of potentiality". Kenny's remark is somewhat unsatisfactory for at least two reasons: In the first place, the formal analysis of modalities is an accomplishment of the possible-worldstheory of possibility that is not to be despised. In the second place, the charge of obliterating the distinction between the potential and the actual can only be raised against certain, by no means generally accepted variants of the possible-worlds-theory of possibility (for example, the conception of David Lewis). Nevertheless Kenny's remark in the context of a book on a medieval philosopher - inspired me to attempt a theory of possibility that does without possible worlds (possible alternatives, possible situations, possible events, possible statesof-affairs etc.), that does without any (mere) possibilia at all, yet distinguishes truth, possibility and necessity, and treats possibility as a nonepistemic, non-syntactic concept. Such a theory has already existed. It is the scholastic theory of powers, propensities and dispositions. It has traditionally been condemned as utterly metaphysical (in the pejorative sense); but compared with the possible-worlds-theory it isn't so badly metaphysical after all, as we shall see.

The propensity-theory of possibility need not and will not be considered as the correct theory of possibility. The greater ontological parsimony of the propensity-theory with respect to possibilia is countered by the wider field of application of the possible-worlds theory. Moreover, possible-worlds-theory and propensity-theory have each their favorite problems, in which each does better than the other. The analysis of counterfactual conditionals is, it seems to me, entirely the domain of the possible-worlds-theory, whereas the propensity-theory has an at least considerable advantage in the field of the analysis of comparative possibility-statements ("that $A$ is the case is more possible than that $B$ is the case"), in the field of the analysis of the distinction between "real" and "merely theoretical" possibility, and in the field of

Erkenntnis 38: 323-341, 1993.

(C) 1993 Kluwer Academic Publishers. Printed in the Netherlands. 
the analysis of disposition-predicates, especially comparative disposition-predicates as for example " $x$ is more breakable than $y$ ".

(2) Let $L_{0}$, a sub-language of $L$, be a two-sorted language of predicate-logic with identity, with variables $x, y, z, \ldots$ for individuals, and variables $f, g, h, \ldots$ for properties of these individuals. $A, B, C, \ldots$ are used to schematically designate sentences of $L_{0}$. " $f(x)$ " of $L_{0}$ expresses that individual $x$ has the property $f$. If $A[x]$ is a predicate of $L_{0}$, then $\lambda x A[x]$ is a singular term of $L_{0}$ that can be substituted for property-variables of $L_{0}$, and for no other variables of $L$ (without damage to wellformedness).

Beside the elementary laws of predicate logic with identity we have (with respect to $L_{0}$ ) the axiom-schema of abstraction

$$
\Vdash \wedge x(\lambda y A[y](x) \equiv A[x]) \quad(x \text { not in } A[y], y \text { not in } A[x])
$$

and the rule of property-identity

$$
\text { If } \Vdash \wedge y(f(y) \equiv g(y)) \text {, then } \Vdash f=g .
$$

The rule of property-identity is weaker than the principle of extensionality: $\Vdash \wedge y(f(y) \equiv g(y)) \supset f=g$; the latter is not valid for properties (in the normal sense), but for sets; while the former is valid for properties, if they are not ultrafine-grained. The principle of extensionality does allow to prove $\Vdash \lambda y(A \wedge y=y)=\lambda y(y=y) \vee \lambda y(A \wedge y=y)=$ $\lambda y(y \neq y)$, while the rule of property-identity does not. (This is to be kept in mind in view of the importance of properties $\lambda y(A \wedge y=y)$ in this paper; see Section 8.) The possibility of interpreting $f, g, \ldots$ as referring to sets, which, indeed, is not excluded by the above principles, can be ruled out by postulating the negation of the principle of extensionality: $\Vdash \bigvee f \bigvee g(\wedge y(f(y) \equiv g(y)) \wedge f \neq g)$.

$L_{0}$ is supposed to be a purely "momentary" and "actual" language; that is, the truth-value of each sentence of $L_{0}$ at a given moment of time depends only on the actual state of the world at that moment (not on earlier or later actual or merely possible states of the world, not on merely possible contemporary alternatives; to occur at a certain moment is not a characteristic of a world-state considered in itself). The reader is reminded that these remarks have a merely clarificatory function, and should not be taken to imply the acceptance of an ontology of world-states.

As has been mentioned, $L_{0}$ is a sub-language of $L . L$ includes in addition variables $t, t^{\prime}, t^{\prime \prime}, \ldots$ taking moments of time as values, a con- 
stant $n$ for now, variables $r, r^{\prime}, r^{\prime \prime}$. . taking real numbers qua quantities as values, and standard names for real numbers qua quantities, moreover standard arithmetical expressions $r+r^{\prime}, r-r^{\prime}, \ldots$ I proceed on the assumption that the moments of time have the same structure as the real numbers qua positions; I therefore identify the moments of time with the real numbers qua positions: $t, t^{\prime}, t^{\prime \prime}$. . refer to real numbers qua positions, $r, r^{\prime}, r^{\prime \prime}$. . to real numbers qua quantities. $t<. t^{\prime}$ means that $t^{\prime}$ is later than $t ; r<r^{\prime}$ means that $r^{\prime}$ is greater than $r$.

In addition $L$ includes a fourplace functional expression that can be substituted for variables that take real numbers qua quantities as values, and for no other variables of $L$ (preserving wellformedness): $\operatorname{pro}\left(x, t, f, t^{\prime}\right)$. The form of an atomic propensity-statement is "pro $\left(x, t, f, t^{\prime}\right)=r$ " or "pro $\left(x, t, f, t^{\prime}\right)<r$ ": "the quantity of the propensity of $x$ at $t$ to be $f$ at $t^{\prime}$ is [is smaller than] $r$ ". Instead of "the quantity of the propensity ..." I simply say "the propensity ...", analogously to saying "the probability..." instead of "the quantity of the probability ...". It has only to be kept in mind that neither propensities nor probabilities are real numbers; they are only measured by them.

(3) For the concept of propensity a heuristic can be formulated in the possible-worlds-theory that yields the same principles as the rather different heuristic I shall actually use below. In order to determine pro $\left(x, t, f, t^{\prime}\right)$ consider the set $P_{t}$ of those possible worlds which coincide with the real world up to (and including) moment $t$ out of a given finite set of nomologically possible worlds including the real world; let $n$ be the cardinal number of $P_{t}$, and $c$ the number of worlds out of $P_{t}$ in which $x$ is $f$ at $t^{\prime}$; we can define $\operatorname{pro}\left(x, t, f, t^{\prime}\right)=c / n$ - and we will obtain the principles listed and discussed below.

Of course the above procedure doesn't capture the concept of propensity (Propensity), but rather one out of many relative concepts of propensity; they are relative to a given finite set of nomologically possible worlds including the real world. Such relative concepts of propensity have structural properties over and above those of Propensity. For example, we have $\bigvee t \wedge x \wedge f \wedge t^{\prime}\left(\operatorname{pro}\left(x, t, f, t^{\prime}\right)=\right.$ $\left.0 \vee \operatorname{pro}\left(x, t, f, t^{\prime}\right)=1\right)$, for sooner or later there will be no more branching of the world-tree because of the finite number of considered nomologically possible worlds.

The assumption of the finiteness of $P_{t}$ is a serious drawback of this heuristic, since this assumption is rather unrealistic indeed (of course, only if nomological determinism is to be rejected, for if nomological 
determinism is right, $P_{t}$ is not only finite for every moment $t$, but consists merely of the real world). As long as $P_{t}$ is countably large, we might attach positive weight (why should a weight be 0 ?) to each world out of $P_{t}$, the sum of these weights being 1 , and consider $\operatorname{pro}\left(x, t, f, t^{\prime}\right)$ to be the sum of the weights of those worlds out of $P_{t}$ in which $x$ is $f$ at $t^{\prime}$. But the question arises in what manner we should attach weights to the worlds out of $P_{i}$; there are uncountably many ways in which this can be done. Unavoidably this leads to a subjectivist conception of propensity, which we, however, have to avoid if we want to use the concept of propensity for the analysis of objective concepts of possibility. Finally, this approach fails when $P_{t}$ is uncountably large (even if we allow non-denumerably many members of the sum: all the weights were supposed to be positive), which, after all, seems to be commonly the case.

Nevertheless it should not go unmentioned that the possible-worlds heuristic allows an intuitively satisfactory justification of a propensityprinciple (i.e. P3) where the other heuristic actually used here is not so intuitively satisfactory.

(4) The concept of Propensity will here be explained by two notions that are well-known from ordinary discourse: distance from realization and difficulty of realization. ${ }^{1}$ Some examples for this: "It's rather difficult for him to reach the 9.30 plane to London", "He is further away from being President this time next year than he was a month ago", "It's easier for me to be at Jim's place at noon, than it is for me to pick you up at the church at 11.45 ". It would be a mistake to interpret these sentences as simply expressing subjective probability judgments. (This seems anyway to be possible only for the first two.) You can be proved to be mistaken about difficulties of realization, you cannot be proved to be mistaken about your own subjective probabilities (lapses of memory aside): This is normal: "He had a helicopter? Well, then it was of course not difficult for him to reach the 9.30 plane to London". But this is deviant: "He had a helicopter? Well, then my probability for his reaching the 9.30 plane to London was of course not low".

The notion of distance from realization is merely a metaphorical variant of the notion of difficulty of realization; its cognitive content coincides with that of the latter. I shall use them interchangeably. These notions allow numerical gradations which have a lower limit: 0 (it makes no sense to speak of negative distances or difficulties), but no upper limit, that is, there is no highest real number that can measure a 
distance from realization or be a degree of difficulty of realization. It may happen in a particular case that no real number greater than or equal to 0 can be assigned measuring distance from realization. If we read $d\left(x, t, f, t^{\prime}\right)$ as "the difficulty for $x$ at $t$ to be $f$ at $t^{\prime}$ " (or as "the distance of $x$ at $t$ from being $f$ at $t^{\prime \prime}$ "), then we can define: $\operatorname{pro}\left(x, t, f, t^{\prime}\right):=1 /\left(d\left(x, t, f, t^{\prime}\right)+1\right)$, in case $d\left(x, t, f, t^{\prime}\right)$ is a real number greater than or equal to 0 ; if it is not a real number greater than or equal to 0 (but rather some arbitrary non-numerical object singled out to give $d\left(x, t, f, t^{\prime}\right)$ an artificial value where this function is initially not defined), then $\operatorname{pro}\left(x, t, f, t^{\prime}\right):=0$.

$d\left(x, t, f, t^{\prime}\right)$ is here understood in such a way that for the determination of its value only the inner and outer determinants operative on $x$ at $t$ are to be considered, not, however, the independent effort (intensio or conatus) $x$ at $t$ puts into being $f$ at $t^{\prime}$ (indeed, there might not be such a thing); in other words, $d\left(x, t, f, t^{\prime}\right)$ is the correlate of what is given to $x$ at $t$ with respect to the realization of $f$ at $t^{\prime}$ (not of what he makes out of what is given to him). (The effort $x$ puts into being $f$ at $t^{\prime}$ at some moment prior to $t$ may well be a determinant operative on $x$ at $t$ which influences $d\left(x, t, f, t^{\prime}\right)$.) This particular way of understanding $d\left(x, t, f, t^{\prime}\right)$ results from the fact that we are not interested in the power of $x$ at $t$ to be $f$ at $t^{\prime}$, but only in the propensity of $x$ at $t$ to be $f$ at $t^{\prime}$ (the former might be quite high while the latter is low; this would be due to the great exertions $x$ undertakes at $t$ to be $f$ at $t^{\prime}$ ).

Difficulty of realization is here treated as a basic functional notion which is not analyzed in terms of a more basic one. It will be argued that we have an intuitive grasp of it and can even determine precise values for it (see Section 7); hence I am justified in leaving it unanalyzed. At first glance it may seem that it can be appropriately analyzed by degree of similarity between possible world-states. This analysis would of course bring in the possible-worlds-theory of possibility by the backdoor; therefore it must not be pursued here, and luckily we need not follow it, since the concept of difficulty of realization is sufficiently clear by itself. But, in fact, this proposed analysis is false. The difficulty for $x$ at $t$ to be $f$ at $t^{\prime}$ may be low, while the state of the world at $t$ is very much dissimilar from any at $t$ possible $t^{\prime}$-state of the world in which $x$ is $f$. Consider a madman $x$ pressing at $t$ the red button, which act determines his end at $t^{\prime}$ in the general nuclear holocaust; the difficulty for $x$ at $t$ to die at $t^{\prime}$ is zero; but any at $t$ possible $t^{\prime}$-state of the world (each one including the annihilation of all higher forms of life) is very 
much dissimilar from the state of the world at $t$. (This argument is an adaptation of an argument by Kit Fine against the Lewis-analysis of counterfactual conditionals employing the notion of comparative similarity of possible worlds.)

(5) From the considerations in Section 4 it can be immediately seen that we are justified in accepting the following principle as an axiom:

$$
\Vdash 0 \leqslant \operatorname{pro}\left(x, t, f, t^{\prime}\right) \leqslant 1 \text {. }
$$

"pro $\left(x, t, f, t^{\prime}\right)=1 "$ means that $d\left(x, t, f, t^{\prime}\right)=0 ; x$ has at $t$ already reached the realization of $f$ at $t^{\prime}$, although, in case $t<. t^{\prime}$, it will take until the coming of $t^{\prime}$ until this realization becomes apparent. "pro $\left(x, t, f, t^{\prime}\right)=0 "$, on the other hand, means that $d\left(x, t, f, t^{\prime}\right)$ is neither equal to zero nor to any positive real number, or, in other words, that the realization of $f$ at $t^{\prime}$ is at $t$ (absolutely) unreachable for $x$.

The property non $(f)$ is $\lambda y \neg f(y)$. The easier it is for $x$ at $t$ to be $f$ at $t^{\prime}$, the more difficult it is for $x$ at $t$ to be non $(f)$ at $t^{\prime}$. The taking into account of the independent effort of the agent for the determination of difficulty of realization, that is, the confusion of propensity with power, is responsible for the illusion that this is not generally true; $x$ may of course have at $t$ a high power to be $f$ at $t^{\prime}$, and an equally high power to be non $(f)$ at $t^{\prime}$; the first would be due to the high inclination of $x$ at $t$ to be $f$ at $t^{\prime}$, the second would be due to the great effort $x$ puts at $t$ into being non $(f)$ at $t^{\prime}$, although his $t$-inclination to be non $(f)$ at $t^{\prime}$ is low proportionally to the highness of the "contradictory" inclination.

In other words, the difficulty for $x$ at $t$ to be non $(f)$ at $t^{\prime}$ is inversely proportional to the difficulty for $x$ at $t$ to be $f$ at $t^{\prime}: d\left(x, t, \operatorname{non}(f), t^{\prime}\right)$ $=1 / d\left(x, t, f, t^{\prime}\right)$, given that $\operatorname{pro}\left(x, t, f, t^{\prime}\right)$ and $\operatorname{pro}\left(x, t, \operatorname{non}(f), t^{\prime}\right)$, $d\left(x, t, f, t^{\prime}\right)$ and $d\left(x, t\right.$, non $\left.(f), t^{\prime}\right)$ differ from 0 . This yields: $\operatorname{pro}\left(x, t, \operatorname{non}(f), t^{\prime}\right)=1-\operatorname{pro}\left(x, t, f, t^{\prime}\right)$ given that $\operatorname{pro}\left(x, t, \operatorname{non}(f), t^{\prime}\right)$, pro $\left(x, t, f, t^{\prime}\right), d\left(x, t, f, t^{\prime}\right)$ and $d\left(x, t, \operatorname{non}(f), t^{\prime}\right)$ differ from 0 . Now: $d\left(x, t, \operatorname{non}(f), t^{\prime}\right)$ is no real number greater than or equal to 0 iff $d\left(x, t, f, t^{\prime}\right)=0 ; d\left(x, t, f, t^{\prime}\right)$ is no real number greater than or equal to 0 iff $d\left(x, t, \operatorname{non}(f), t^{\prime}\right)=0$. This yields: $\operatorname{pro}\left(x, t, \operatorname{non}(f), t^{\prime}\right)=$ $1-\operatorname{pro}\left(x, t, f, t^{\prime}\right)$, given that $\operatorname{pro}\left(x, t, \operatorname{non}(f), t^{\prime}\right)$ or $\operatorname{pro}\left(x, t, f, t^{\prime}\right)$ or $d\left(x, t, f, t^{\prime}\right)$ or $d\left(x, t, \operatorname{non}(f), t^{\prime}\right)$ is 0 . These considerations justify us in assuming as an axiom:

$$
\Vdash \operatorname{pro}\left(x, t, \operatorname{non}(f), t^{\prime}\right)=1-\operatorname{pro}\left(x, t, f, t^{\prime}\right) .
$$


The property $(f \cup g)$ is $\lambda y(f(y) \vee g(y))$, and the property $(f \cap g)$ is $\lambda y(f(y) \wedge g(y))$. I postulate as axioms:

P3

$$
\begin{aligned}
\Vdash \operatorname{pro}\left(x, t,(f \cup g), t^{\prime}\right)= & \operatorname{pro}\left(x, t, f, t^{\prime}\right)+\operatorname{pro}\left(x, t, g, t^{\prime}\right), \\
& -\operatorname{pro}\left(x, t,(f \cap g), t^{\prime}\right),
\end{aligned}
$$

P4

$$
\Vdash \operatorname{pro}\left(x, t,(f \cap \operatorname{non}(f)), t^{\prime}\right)=0 .
$$

Like the principles P1 and P2, P3 and P4 correspond to well-known probabilistic principles. (No surprise: the propensity-definition of probability is a viable alternative to other definitions of probability. ${ }^{2}$ ) The rationale for $\mathrm{P} 4$ in terms of the distance-heuristic is clear: $d(x$, $\left.t,(f \cap \operatorname{non}(f)), t^{\prime}\right)$ is no real number greater than or equal to zero, whatever $x, t, t^{\prime}$, or $f$ may be. But $\mathrm{P} 3$ throws more light on the distanceheuristic than the distance-heuristic throws on P3. Here we can use with advantage the possible-worlds-heuristic introduced in Section 3, which gives a straightforward intuitive justification for P3.

(6) I now come to principles for Propensity that have no probabilistic analogues. Consider first the propensity of $x$ at $t$ to be $f$ at the very same moment $t$. What sizes can be taken on by this propensity? One easily sees that there are only two possibilities; for either $x$ is $f$ at $t$, or $x$ is not $f$ at $t$; in the former case being non $(f)$ at $t$ is unreachable for $x$ at $t$, that is, $d(x, t, \operatorname{non}(f), t)$ is no real number greater than or equal to 0 , that is, $\operatorname{pro}(x, t, \operatorname{non}(f), t)=0$, that is, $\operatorname{pro}(x, t, f, t)=1$; in the latter case being $f$ at $t$ is unreachable for $x$ at $t$, that is, $\operatorname{pro}(x, t, f, t)=$ 0 . I accordingly postulate as an axiom:

P5

$$
\Vdash \operatorname{pro}(x, t, f, t)=1 \vee \operatorname{pro}(x, t, f, t)=0 .
$$

Propensities can change in time. The distance of $x$ from the realization of $f$ at $t^{\prime}$ can become greater and smaller; but only as long as $x$ does not arrive at the realization of $f$ at $t^{\prime}$. If it does arrive there, it has to stay there; a realization cannot be taken back. Hence we have as an axiom:

P6

$$
\Vdash \operatorname{pro}\left(x, t, f, t^{\prime}\right)=1 \supset \bigwedge t^{\prime \prime}\left(t<. t^{\prime \prime} \supset \operatorname{pro}\left(x, t^{\prime \prime}, f, t^{\prime}\right)=1\right) .
$$

The following axiom will not be used for the purposes of this paper. It is included in order to show that the concept of Propensity is interesting to be investigated independent of the considerations this paper is limited to.

It can be easily seen that $\bigwedge x \wedge z(f(x) \equiv \lambda y(f(x) \wedge y=y)(z))$ is pro- 
vable. What is in view of this fact the relation between $\operatorname{pro}\left(x, t, f, t^{\prime}\right)$ and $\operatorname{pro}\left(z, t, \lambda y(f(x) \wedge y=y), t^{\prime}\right)$ ? Let us for the moment - presystematically - introduce propensities whose objects are not properties, but rather states-of-affairs. "pro $\left(t, p, t^{\prime}\right)=r$ " means, that the propensity at $t$ that the state-of-affair $p$ is realized at $t$ ' is equal to $r$. (Singular terms referring to states-of-affairs can be obtained from any sentence of $L_{0}$ by prefixing "that" to it.) The provable general equivalence mentioned above shows that in every case the state-of-affair that $x$ is $f$ is identical to the state-of-affair that $z$ is self-identical, while $x$ is $f$. Hence we have in general:

$$
\text { pro }\left(t, \text { that } f(x), t^{\prime}\right)=\operatorname{pro}\left(t, \text { that } \lambda y(f(x) \wedge y=y)(z), t^{\prime}\right) \text {. }
$$

Now evidently $\operatorname{pro}\left(x, t, f, t^{\prime}\right)=\operatorname{pro}\left(t\right.$, that $\left.f(x), t^{\prime}\right)$ and $\operatorname{pro}(z, t$, $\left.\lambda y(f(x) \wedge y=y), t^{\prime}\right)=\operatorname{pro}\left(t\right.$, that $\left.\lambda y(f(x) \wedge y=y)(z), t^{\prime}\right)$. Hence we are justified in accepting as an axiom:

P7 $\Vdash \operatorname{pro}\left(x, t, f, t^{\prime}\right)=\operatorname{pro}\left(z, t, \lambda y(f(x) \wedge y=y), t^{\prime}\right)$

For the time being I conclude the list of postulates (which is by no means complete; the complete list of axioms might, for example, include an axiom that states that propensities change continually in time $\mathrm{e}^{3}$ ). Before I will use the concept of Propensity for the explication of other concepts, I introduce a model that visualizes the matter of fact described by the above principles, and I give an example of the determination of the precise numerical value for $d\left(x, t, f, t^{\prime}\right)$, respectively $\operatorname{pro}\left(x, t, f, t^{\prime}\right)$.

(7) Let every straight line parallel to $g$ represent a property; let every straight line parallel to $t$ represent a moment of time:

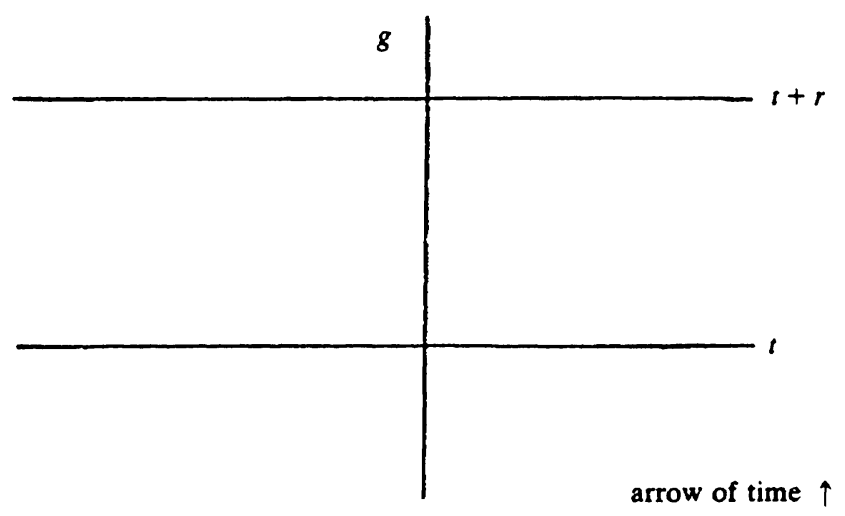


Above the plane partitioned in this manner lies a (porous, at most onepoint thick) blanket of points which are in part in vertical motion (relative to the plane). Consider this blanket in the phase $t$. For all points of the plane which are lower than or on the $t$-line we have that the blanket is attached to them or has a hole at their place. Holes in the blanket will never close once they have appeared, nor will the blanket ever disengage itself from any point of the plane it has once become attached to. In the part of the plane which is above the $t$-line we have, besides the cases of attachment and porosity of the blanket, a third case: some points of the blanket have a finite vertical distance to the plane; with respect to the part of the plane above the $t$-line the blanket is partly in suspension. If we observe the movement of the blanket during the interval $r$ we will see that the blanket increasingly leaves its state of partial suspension. Consider it in the phase $t+r$. Then it is true for all points of the plane which are below or on the $t+r$-line that the blanket is either attached to them or has a hole at their place. And for the points of the plane above the $t+r$-line it is true that there are now more (or at least no less) among them to which the blanket is attached or where it has a hole than there were when the blanket had been in the phase $t$. (Where the blanket was previously attached there it still is, and where the blanket previously had a hole there it still has one). If there were a final time-line, an ultimate state would be reached when the blanket is in the phase corresponding to that time-line where for every point of the plane it is true that the blanket attaches to it or has a hole at its place.

The blanket of points above the plane represents an indeterministic individual $x$. For every phase $t$ of the blanket $x$ there is a complete description of the function $d\left(x, t, f, t^{\prime}\right)$. Consider the blanket in such a phase and consider the point of intersection of the $t^{\prime}$-line and the $f$ line: $\left(f, t^{\prime}\right)$. If $x$ has a hole at $\left(f, t^{\prime}\right)$, then $d\left(x, t, f, t^{\prime}\right)$ is no real number greater than or equal to 0 , but, say, Napoleon. If $x$ is attached to $\left(f, t^{\prime}\right)$, then $d\left(x, t, f, t^{\prime}\right)=0$. If there is a point of the blanket vertically above $\left(f, t^{\prime}\right)$ (it is at most one), then $d\left(x, t, f, t^{\prime}\right)=r$, where $r$ is the distance of that point from the plane.

The positions of the points of the blanket are not in every case independent of each other in a certain phase of the movement of the blanket. If $p$ is a point of the blanket vertically above $\left(f, t^{\prime}\right)$ at the positive distance $r$, then there is a point of the blanket $p^{\prime}$ vertically above $\left(\operatorname{non}(f), t^{\prime}\right)$ at the distance $1 / r$; if the blanket attaches to $\left(f, t^{\prime}\right)$, 
then it has a hole at $\left(\operatorname{non}(f), t^{\prime}\right)$; if it has a hole at $\left(f, t^{\prime}\right)$, then it attaches to $\left(\operatorname{non}(f), t^{\prime}\right)$. If $f$ and $g$ are properties which exclude each other [their conjunction is identical to $(f \cap \operatorname{non}(f))$ ], and if there is a point $p^{\prime}$ of the blanket having the vertical distance $r$ from $\left(f, t^{\prime}\right)$ and a point $p^{\prime \prime}$ of the blanket having the vertical distance $r^{\prime}$ from $\left(g, t^{\prime}\right)$, then there is a point $p^{\prime \prime \prime}$ of the blanket having the vertical distance $\left(r \cdot r^{\prime}-1\right) /\left(r+r^{\prime}+2\right)$ from $\left((f \cup g), t^{\prime}\right)$; and so on in the appropriate manner for the cases not yet considered. Moreover, we have at every point $\left((f \cap \operatorname{non}(f)), t^{\prime}\right)$ of the plane (no matter what property $f$ is or what moment $\left.t^{\prime}\right)$ that the blanket has a hole there - no matter what phase the blanket is in.

The description of this visual model is sufficient for the verification of the principles $\mathrm{P} 1$ to $\mathrm{P} 6$, given the heuristic definition of $\operatorname{pro}\left(x, t, f, t^{\prime}\right)$ by $d\left(x, t, f, t^{\prime}\right)$. Significantly, there is no reference to any entities in the model that might be taken to represent possible alternatives ("worlds"). The propensity-theory of possibility can rightfully be called an "immanent theory of possibility", since the only possible world it needs to take into account is the real world; it stays within the limits of this world (without thereby identifying truth and - objective - necessity!).

The determination of the precise numerical value of $d\left(x, t, f, t^{\prime}\right)$ and hence of $\operatorname{pro}\left(x, t, f, t^{\prime}\right)$ in a concrete case is difficult because there are so many factors that have to be considered for this. But it is not difficult to make accurate comparative non-numerical judgments of distance from (difficulty of) realization. Everyone of us does this. It would be unexplainable why we persist in this practice, if there were no objective fact of the matter referred to by these statements or if these statements were uncontrollable for us. Hence there are such things as distances from realization, and we are able to recognize them.

In certain more or less artificial cases the numerical value of $d\left(x, t, f, t^{\prime}\right)$, respectively $\operatorname{pro}\left(x, t, f, t^{\prime}\right)$ can be precisely determined. Let $x$ be a materially homogeneous die here on earth thrown straight into the air at $t$ above a hard and plane surface of sufficient extent, and let it be excluded at $t$ that the die is caught by somebody in mid air or manipulated, that it explodes in mid air, vanishes or anything; let it be excluded at $t$ that the surface is removed, softened or deformed while the die is flying and for some time longer; then there is a moment $t^{\prime}$ later than $t$, such that the propensity of $x$ at $t$ to lie on the surface at $t^{\prime}$ showing one of its six sides uppermost is 1 , and such that the propensity of $x$ at $t$ to lie on the surface at $t^{\prime}$ showing, for example, 5 uppermost 
is $1 / 6$. (The value $1 / 6$ for the latter propensity can be deduced from the value 1 for the former propensity by applying the principles above, and by making use both of the fact that the die can show only one of its six sides uppermost [the property of showing its side $n$ uppermost and the property of showing its side $k$ uppermost exclude each other for $n \neq k]$ and of the fact that the arrangement guarantees that no side is preferred - at $t$ ! - to any non-negligible degree. ${ }^{4} \operatorname{pro}(x, t$, showing $\left.5, t^{\prime}\right)=1 / 6$ does in no manner preclude that $\operatorname{pro}\left(x, t^{+}\right.$, showing $\left.5, t^{\prime}\right)=1$ for some $t^{+}, t<. t^{+}<. t^{\prime}$, which means that the die's showing 5 at $t^{\prime}$ was inevitable prior to $t^{\prime}$; it is compatible with a species of determinism that assumes the determination of the present by the past, but leaves open just at what time each item of the present is determined; the time may be different for some or all items.)

$$
\begin{array}{ll}
\text { D1 } & \begin{array}{l}
P\left(x, t, f, t^{\prime}\right): \equiv \operatorname{pro}\left(x, t, f, t^{\prime}\right) \neq 0 \\
\left(\text { it is possible for } x \text { at } t \text { to be } f \text { at } t^{\prime}\right),
\end{array} \\
\text { D2 } \quad \begin{array}{l}
N\left(x, t, f, t^{\prime}\right): \equiv \operatorname{pro}\left(x, t, f, t^{\prime}\right)=1 \\
\end{array} \\
\text { (it is necessary for } \left.x \text { at } t \text { to be } f \text { at } t^{\prime}\right),{ }^{5} \\
\text { D3 } \quad T(x, f, t): \equiv \operatorname{pro}(x, t, f, t)=1 \\
& (x \text { is f at } t) .
\end{array}
$$

Then we have the following theorems:

$$
\begin{aligned}
& \Vdash N\left(x, t, f, t^{\prime}\right) \equiv \neg P\left(x, t, \operatorname{non}(f), t^{\prime}\right), \\
& \Vdash T(x, f, t) \equiv \neg T(x, \operatorname{non}(f), t), \\
& \Vdash T(x, f, t) \equiv N(x, t, f, t) \equiv P(x, t, f, t) .
\end{aligned}
$$

T3 will shortly be commented on. The step from the three- or fourplace predicates introduced in D1-D3 to sentence-connectives is made in the next triad of definitions. The iteration and interlacing of these connectives is syntactically forbidden, since for substitution into their scope only sentences of $L_{0}$ are admitted, and every sentence containing one of these connectives is not a sentence of $L_{0}$ (but only of $L$ ). This restriction is not a must; solely for reasons of problem-reduction I exclusively consider propensities which have as their objects "manifest" and "momentary" properties (properties whose exemplification or nonexemplification by an individual at a certain moment depends only on 
the actual state of the world at that moment); this precisely has the consequence that only sentences of $L_{0}$ can be substituted into the scope of the connectives to be introduced now:

D4 $P\left(t, A, t^{\prime}\right): \equiv \bigvee x P\left(x, t, \lambda y(A \wedge y=y), t^{\prime}\right)(x, y$ not in $A)$ (it is possible at $t$ that it is the case at $t^{\prime}$ that $A$ ),

D5 $\quad N\left(t, A, t^{\prime}\right): \equiv \bigwedge x N\left(x, t, \lambda y(A \wedge y=y), t^{\prime}\right)(x, y$ not in $A)$, (it is necessary at $t$ that it is the case at $t^{\prime}$ that $A$ ),

D6

$$
T(t, A): \equiv \wedge x T(x, \lambda y(A \wedge y=y), t)
$$

(it is the case at $t$ that $A$ ).

We have the following theorems which show the adequacy of these definitions for introducing alethic modal operators:

$\mathrm{T} 4$

$$
\Vdash N\left(t, A, t^{\prime}\right) \equiv \neg P\left(t, \neg A, t^{\prime}\right),
$$

T5

$$
\Vdash N\left(t, A, t^{\prime}\right) \supset T\left(t^{\prime}, A\right),
$$

T6

$$
\Vdash N\left(t, A, t^{\prime}\right) \wedge N\left(t, A \supset B, t^{\prime}\right) \supset N\left(t, B, t^{\prime}\right),
$$

T7 If $\Vdash A$, then $\Vdash N\left(t, A, t^{\prime}\right)$.

(For the proof of T6 I have used the following two other theorems:

$$
\begin{aligned}
& \text { I } \operatorname{pro}\left(x, t, f, t^{\prime}\right)=1 \wedge \operatorname{pro}\left(x, t, g, t^{\prime}\right)=1 \\
& \supset \operatorname{pro}\left(x, t,(f \cap g), t^{\prime}\right)=1, \\
& \text { \& } \operatorname{pro}\left(x, t, f, t^{\prime}\right)=r \wedge \operatorname{pro}\left(x, t,(f \cap \operatorname{non}(g)), t^{\prime}\right)=0 \\
& \left.\supset r \leqslant \operatorname{pro}\left(x, t, g, t^{\prime}\right) .\right)
\end{aligned}
$$

The next theorem shows as much as $\mathrm{T} 3$ that the alethic modalities introduced by D1-D6 are special ones:

$\mathrm{T} 8$

$$
\Vdash T(t, A) \equiv N(t, A, t) \equiv P(t, A, t) .
$$

T3 and T8 can best be understood intuitively by considering that the concept of necessity captured by D5 and D2 is that of unchangeability. If it is the case at $t$ that $A$, then it is unchangeable at $t$ that it is the case at $t$ that $A$; if $x$ is $f$ at $t$, then being $f$ at $t$ is unchangeable for $x$ at t. This intuitively justifies $T(t, A) \supset N(t, A, t)$ and $T(x, f, t) \supset$ $N(x, t, f, t)$. (For $\Vdash P(t, A, t) \supset T(t, A): \Vdash T(t, \neg A) \supset N(t, \neg A, t)$, hence $\Vdash \neg N(t, \neg A, t) \supset \neg T(t, \neg A)$, hence $\Vdash P(t, A, t) \supset T(t, A)$ by T4 and $\Vdash T(t, A) \equiv \neg T(t, \neg A)$ which is provable using T2.)

T4-T8 are sentential principles for modalities. A quantificational 
principle is for example the converse of the Barcan-formula: $N\left(t, \bigwedge x A[x], t^{\prime}\right) \supset \bigwedge x N\left(t, A[x], t^{\prime}\right)$, which is provable without extra axioms. The Barcan-formula itself: $\bigwedge x N\left(t, A[x], t^{\prime}\right) \supset N\left(t, \bigwedge x A[x], t^{\prime}\right)$ is uncontroversial within the propensity-theory of possibility, since reference to various possible worlds with their respective universes of discourse is out of the question. But the formula is not provable without additional axioms. I sketch its proof within an extended axiom-system: The great conjunction of all properties $f$ such that $\bigvee x(f=$ $\left.\lambda y^{\prime}\left(A[x] \wedge y^{\prime}=y^{\prime}\right)\right), \rho$, is identical with $\lambda y^{\prime}\left(\bigwedge x A[x] \wedge y^{\prime}=y^{\prime}\right)$; consequently $\quad \operatorname{pro}\left(y, t, \rho, t^{\prime}\right)=1 \supset \operatorname{pro}\left(y, t, \lambda y^{\prime}\left(\bigwedge x A[x] \wedge y^{\prime}=y^{\prime}\right)\right.$, $\left.t^{\prime}\right)=1$; but $\operatorname{pro}\left(y, t, \rho, t^{\prime}\right)=1$ iff $\bigwedge x\left(\operatorname{pro}\left(y, t, \lambda y^{\prime}\left(A[x] \wedge y^{\prime}=y^{\prime}\right)\right.\right.$, $\left.\left.t^{\prime}\right)=1\right) ; \quad$ hence $\quad \wedge x \wedge y\left(\operatorname{pro}\left(y, t, \lambda y^{\prime}\left(A[x] \wedge y^{\prime}=y^{\prime}\right), t^{\prime}\right)=1\right) \supset$ $\wedge y\left(\operatorname{pro}\left(y, t, \lambda y^{\prime}\left(\bigwedge x A[x] \wedge y^{\prime}=y^{\prime}\right), t^{\prime}\right)=1\right)$, hence by D5 the Barcanformula. ${ }^{6}$

(9) The usual appearance of modal connectives is, for example, reached by the following definition which interprets "it is necessary that $A$ " as meaning the same as "it is now already unchangeably fixed that it will be the case that $A$ ":

D7

$$
N^{*} A: \equiv \bigvee t(n<. t \wedge N(n, A, t))
$$

Compare this definition with the definition of "it will be the case that A":

D8 $F A:=\bigvee t(n<. t \wedge T(t, A))$.

It can easily be proved:

$$
\Vdash N^{*} A \supset F A \text {. }
$$

The reverse of T9 cannot be proved. But there is some possibility of confusing $N^{*} A$ and $F A$. Consider that $T(t, A)$ in the definition of $F A$ is synonymous with $N(t, A, t)$, and consider that the phrase "there is a later time at which it is necessary that $A$ " can be read both in the sense of "there is a later time such that it is now necessary that it is the case at it that $A^{\prime \prime}\left(N^{*} A\right)$, and in the sense of "there is a later time such that it is necessary at it that it is the case that $A$ " $(F A)$. This confusion may be one of the reasons underlying attempts to "prove" determinism with respect to the future.

The sentential connective $N^{+}$is directed in the reverse temporal direction of $N^{*}$ : 
D9

$$
N^{+} A: \equiv \bigvee t(t \leqslant . n \wedge N(n, A, t)) .
$$

D9 gives "it is necessary that $A$ " a different sense than D7 does, namely the sense of "it is now unchangeably fixed that it was or is the case that $A$ ". Not surprisingly we can prove

T10 $\Vdash N^{+} A \equiv P A \vee A$.

if we define $P A$ by

D10 $P A: \equiv \bigvee t(t<. n \wedge T(t, A))$,

and add to our list of postulates

P8 $\Vdash A \equiv T(n, A)$.

(P8 sanctions substitution of $T(n, A)$ for $A$ only where this is syntactically possible; the following is incorrect not merely because the inference is not valid, but because it leads to an ill-formed expression: " $N\left(t, A, t^{\prime}\right)$, hence $N\left(t, T(n, A), t^{\prime}\right)$ ".)

The formula $F A \supset N^{*} A$ expresses a version of determinism that might be termed "forward-determinism"; according to forward-determinism the future is fixed by the present. "Backward-determinism" is expressed by $A \supset \bigvee t^{\prime}\left(t^{\prime}<. n \wedge N\left(t^{\prime}, A, n\right)\right)$; according to backwarddeterminism the present is fixed by the past. Of course, concerning determinism there seems to be nothing special about the present; therefore, whoever accepts backward- or forward-determinism will presumably also accept the more general deterministic hypotheses $\bigwedge t\left(T(t, A) \supset \bigvee t^{\prime}\left(t^{\prime}<. t \wedge N\left(t^{\prime}, A, t\right)\right)\right)$ or $\wedge t\left(\bigvee t^{\prime}\left(t<. t^{\prime} \wedge T\left(t^{\prime}, A\right)\right)\right.$ $\left.\supset \bigvee t^{\prime}\left(t<. t^{\prime} \wedge N\left(t, A, t^{\prime}\right)\right)\right){ }^{7}$

By defining another necessity-operator $N^{\#}$ we can express backwarddeterminism - just like forward-determinism - without explicit reference to moments of time by $A \supset N^{\#} A$ :

D11 $N^{*} A: \equiv \bigvee t(t<. n \wedge N(t, A, n))$.

(Compare definitions D9, D10 and D11!) D11 interprets "it is necessary that $A$ " as "it was unchangeably fixed that it is now the case that $A$ ".

(10) Finally I would like to show the use of the concept of Propensity for the analysis of disposition predicates. As examples I will pick the predicates " $x$ is inflammable" and " $x$ is at least as inflammable as $y$ ". I will designate the property expressed by the manifest predicate " $x$ is burning" by " $b$ ". There are uses of " $x$ is inflammable" in which it 
implies " $x$ is not burning"; there are others in which it is implied by " $x$ is burning" (you cannot have both, or else you will be able to prove that nothing burns). I select " $x$ is inflammable" in the second sense for analysis:

$$
x \text { is (at all) inflammable }: \equiv \bigvee_{t}(n \leqslant . t \wedge \operatorname{pro}(x, n, b, t)>0) .
$$

This is " $x$ is inflammable" in a very weak sense. We can give it a very strong sense:

$$
x \text { is (very) inflammable }: \equiv \bigvee t(n \leqslant . t \wedge \operatorname{pro}(x, n, b, t)>0.9) .
$$

Concerning " $x$ is at least as inflammable as $y$ ", the basic analysis is:

$$
\begin{aligned}
& x \text { is (always from now on) at least as inflammable as } \\
& y: \equiv \wedge t(n \leqslant . t \supset \operatorname{pro}(y, n, b, t) \leqslant \operatorname{pro}(x, n, b, t)) .
\end{aligned}
$$

But normally one doesn't mean to assert something as strong as this by saying " $x$ is at least as inflammable as $y$ "; rather one is tacitly and more or less vaguely referring to a certain time interval $\left[n, t_{0}\right]$, and means to say that $x$ is at least as inflammable as $y$ in this interval ( $t_{0}$ is normally rather close to $n$ ):

$$
\begin{aligned}
& \left.x \text { is (up to } t_{0}\right) \text { at least as inflammable as } y: \equiv \bigwedge t(n \leqslant t \\
& \left.\leqslant . t_{0} \supset \operatorname{pro}(y, n, b, t) \leqslant \operatorname{pro}(x, n, b, t)\right) .
\end{aligned}
$$

The definitions (1) and (2) above can be modified with the same justification in the same manner (3) has been modified in ( $\left.3^{\prime}\right)$; this yields definitions $\left(1^{\prime}\right)$ and $\left(2^{\prime}\right)$. Any analysis of " $x$ is at least as inflammable as $y$ " and " $y$ is inflammable" ought to justify the following inference: " $y$ is inflammable, $x$ is at least as inflammable as $y \rightarrow x$ is inflammable". This inference is justified on the basis of the definitions (1) and (3), (2) and $(3),\left(1^{\prime}\right)$ and $\left(3^{\prime}\right)$, and $\left(2^{\prime}\right)$ and $\left(3^{\prime}\right)$.

The orthodox analysis of disposition-predicates by conditionals is in some cases apparently false: Let $x$ be an atom of a certain newly formed radioactive substance. We would certainly say that $x$ is decayable. But do we mean by this that if $x$ were subjected to certain conditions (at an arbitrary time $t, n \leqslant . t$ ) it would invariably decay? It seems not: there are no such conditions known; hence we cannot be certain that if $x$ were subjected to certain conditions it would decay; but we are nonetheless certain that $x$ is decayable. Rather, by " $x$ is decayable" we mean that $\bigvee t(n \leqslant . t \wedge \operatorname{pro}(x, n, d, t)>0)$, and we can be more specific than that: $\operatorname{pro}(x, n, d, n+\tau)=1 / 2, \operatorname{pro}(x, n, d, n+2 \tau)=3 / 4, \operatorname{pro}(x$, 
$n, d, n+3 \tau)=7 / 8, \ldots, \tau$ being the radioactive period of the (newly formed) substance, $x$ is belonging to. ${ }^{8}$

A short notice concerning "real" vs. "merely theoretical" possibility. The distinction between real and merely theoretical possibility is wellrooted in ordinary language. For the propensity-theory of possibility there is no difficulty in reconstructing this distinction: it is "really" possible for $x$ at $t$ to be $f$ at $t^{\prime}$ iff $\operatorname{pro}\left(x, t, f, t^{\prime}\right)>0.5$; it is "merely theoretically" possible for $x$ at $t$ to be $f$ at $t^{\prime}$ iff $0<\operatorname{pro}\left(x, t, f, t^{\prime}\right) \leqslant 0.5$.

\section{NOTES}

${ }^{1}$ The connection between difficulty (or ease) of realization, (objective) possibility, and objective probability is an old one, going at least as far back as Leibniz. Ian Hacking writes in [71], p. 602: "In the early days of probability the word 'possibility" did not mean the epistemic or subjective notion commonly associated with Laplace. When Leibniz says, "probability is degree of possibility", it is clear from the context that 'possibility' itself is a synonym for the ease of making an outcome, that is, for the physical property we call 'propensity' or 'tendency to occur'. Far from being idiosyncratic, this usage was widespread. Indeed, although in his more philosophical writings Laplace professes to assign an epistemic use to 'possibility', in his most important work on the probability of causes even Laplace uses 'possibility' to mean objective propensity". Hacking continues: "Leibniz used 'equally possible' to mean something like 'having an equal propensity to occur'. $\mathrm{He}$ is also the first to use 'possibility' for a quite different idea which, for today's logician, has superseded every other. 'Possible', according to him means internally consistent". While Hacking is right (at least prima facie) in distinguishing two concepts of possibility (one admitting of degrees, the other not) and in noting the current eclipse of one of these, he is wrong in ascribing their discovery to Leibniz. The medievals were very well aware of the distinction: "Possibile autem fuit ens creatum esse, antequam esset per potentiam agentis, per quam et esse incoepit. Vel propter habitudinem terminorum in quibus nulla repugnantia invenitur: quod quidem possibile secundum nullam potentiam dicitur" (Thomas Aquinas, Summa contra Gentiles, 2, 37).

${ }^{2} \mathrm{P}$. Humphreys argues in [85], p. 558f, that conditional propensities cannot be identified with conditional probabilities, since the latter satisfy Bayes' Law [for $p(A), p(B)>0$ : $p(A / B)=(p(B / A) \cdot p(A)) / p(B)]$, while the former do not. But if we define $\operatorname{pro}\left(x, t, f / g, t^{\prime}\right):=\operatorname{pro}\left(x, t,(f \cap g), t^{\prime}\right): \operatorname{pro}\left(x, t, g, t^{\prime}\right)$, provided $\operatorname{pro}\left(x, t, g, t^{\prime}\right) \neq 0$; then the probabilistic principles P1-P4 are sufficient for deducing the analogue of Bayes' Law, and these principles are intuitively well-founded for propensities as representing difficulties of realization. If propensities are understood in this sense, which is not idiosyncratic - see note 1 - then there is no need to pay the high price of changing the calculus of probability (what Humphreys thinks ought to be done; see [85], p. 557) in order to identify objective probability and propensity.

${ }^{3}$ This implies: For any $x, f, t$ and $t^{\prime}, t<. t^{\prime}$, there is inbetween $t$ and $t^{\prime}$ a continuous graph that reaches the value 1 or 0 at $t^{\prime}$ at the very latest, and continues constantly after 
having once reached 1 or 0 . This graph is called "the realization-curve for $x$ and $f$ from $t$ to $t^{\prime}$ ". Here an example of such a realization-curve:

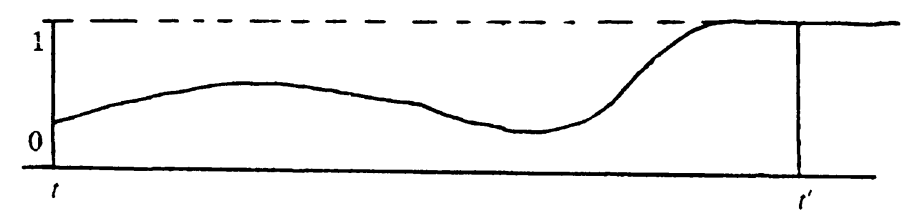

${ }^{4}$ M. C. Galavotti states in [87], p. 363: "single-case propensity theories of probability show severe difficulties with respect to applicability. Various problems of this kind were already stressed by H. Kyburg in 1974, when he pointed out that, in spite of statements to the effect that frequencies do not define propensities, the only method the advocates of propensity theory have at their disposal in order to assign a value to a dispositional strength, and to test it, is that of relying on frequencies". My example ought to show, (1) that Kyburg is not right, (2) that the difficulties for the applicability of single-case propensity theories (while not to be underestimated) can at least in some cases be overcome.

5 A definition with some similarity to this one is given in Giere [79], p. 444: "We will assume, then, that expressions of the form $\operatorname{Pr}_{A}(X)=r$ refer to the propensity of some system in initial state $A$ to reach a final state in $X$ on some particular trial or sets of trials. Utilizing the basic intuition that a propensity of one should correspond to causal necessity, we can quite plausibly define a notion of system necessity for stochastic systems in terms of unit propensities as follows:

(SNS) $\operatorname{Nec}(X)={ }_{\text {Df. }}(A) \operatorname{Pr}_{A}(X)=1 "$.

But Giere is quantifying over possible initial states, which is avoided here. If $y$ is the system referred to, $A$ its actual initial state, $t$ the initial time ( $A$ is the state $y$ is in at $t$ ), $t+r$ the time of the system's final state; then we have: $\operatorname{Pr}_{A}(X)=1$ iff $\operatorname{pro}(y, t, X, t+r)=$ 1.

${ }^{6}$ Smokler writes in [79], p. 503: "The acceptance of de re modalities signifies that one accepts the existence of possible worlds and possible individuals (some of which are actual) which possess necessary properties. This is the ontology which it seems to me the acceptance of single-case probabilities forces upon us". An irreducible de re modal statement that is well-formed in $L$ is, for example, any $L$-sentence of the form $\bigvee x N\left(t, A[x], t^{\prime}\right)$. The acceptance of such a sentence as true does of course - as should be clear by now - not imply that there are possible worlds or possible individuals (other than real ones), and neither does the acceptance of single-case probabilities, at least if they are identified with propensities. With one proviso: that the notion of property (as a kind of intensional entity) is clear without recourse to (mere) possibilia (in particular, that no property is a mere possibilium itself). This is not uncontroversial. For D. Lewis, for example, properties are best identified with sets of possible individuals (including non-actual ones). See Lewis [86]; but consider Armstrong [78] and Bealer [82] where (although in quite different settings) the conception of properties as basic non-possibilistic entities is carefully stated and ably defined. 
${ }^{7}$ M. C. Galavotti says in [87], p. 363: "A direct philosophical shortcoming of propensity theory is that it involves a commitment to indeterminism". This may be true of other propensity theories, it is not true of the propensity theory developed in this paper. Determinism in its strongest version simply means that the value of every propensity (which is always the propensity of some object at some time to have some property at the same or another time) is either 1 or 0 . It does not follow from the propensity theory given here that this kind of determinism does not obtain. Hence it does not follow from it that any weaker version of determinism does not obtain, specifically such a version of determinism that allows some propensities to have a value greater than 0 and less than 1 , as for example $\bigwedge t\left(T(t, A) \supset \bigvee t^{\prime}\left(t^{\prime}<. t \wedge N\left(t^{\prime}, A, t\right)\right)\right)$ : according to this statement there may be, given $T(t, A)$, infinitely many times $t^{\prime \prime}$ prior to $t$ at which for some $x$ $0<\operatorname{pro}\left(x, t^{\prime \prime}, \lambda y(A \wedge y=y), t\right)<1$, provided there is a time $t^{\prime}$ prior to $t$ at which for all $x \operatorname{pro}\left(x, t^{\prime}, \lambda y(A \wedge y=y), t\right)=1$. (By the way, we have $\Vdash \wedge x \wedge z(\operatorname{pro}(x, t, \lambda y(A \wedge y=$ $\left.\left.y), t^{\prime}\right)=\operatorname{pro}\left(z, t, \lambda y(A \wedge y=y), t^{\prime}\right)\right)$, using $\mathrm{P} 7$ and $\Vdash \lambda y(A \wedge y=y)=\lambda y^{\prime}(\lambda y(A \wedge y=$ $\left.y)(x) \wedge y^{\prime}=y^{\prime}\right)$.)

${ }^{8}$ In [79], p. 448, Giere argues as follows: "If we regard time as continuous, which seems to be standard scientific practice, then according to our standard measure theory, each instant of time has zero probability of being the time of decay. But if the atom decays at all, then at least one time is physically possible. So we must give up the identification of physical necessity with unit propensity - at least for systems with non-denumerable outcome sets". To make this more explicit: Assume necessity is unit propensity, hence impossibility is zero propensity, and possibility is non-zero propensity; for any $t$ : the probability (as of now) that $x$ decays at $t$ is zero, hence, since probability = propensity, for any $t$ : the propensity (as of now) that $x$ decays at $t$ is zero, hence for all $t$ : it is (now) impossible that $x$ decays at $t$; assume $x$ decays at some time; hence for some time $t$ : it is (now) possible that $x$ decays at $t$-contradiction. But how does Giere get the idea that for any $t$ : the probability that $x$ decays at $t$ is zero? "The sum of pro $(x, n, f, t)$ over all $t$ is smaller than or equal to $1^{\prime \prime}$ and $\bigwedge t \wedge t^{\prime}\left(\operatorname{pro}(x, n, f, t)=\operatorname{pro}\left(x, n, f, t^{\prime}\right)\right)$ yield of course, given that time is continuous, $\bigwedge t(\operatorname{pro}(x, n, f, t)=0)$. But why should we assume these principles, both of which are glaringly false? (Assume pro $(x, n, f, n)=1-x$ is now $f$ - and $\left.\operatorname{pro}\left(x, n, f, t^{\prime}\right)=1 / 2\right)$.

But might there not be a real problem concerning continuity? Space is a continuum. Hence there are non-denumerably many locations $l$, hence non-denumerably many properties being-at-l, being-at- $l^{\prime}$, which all exclude each other given $l \neq l^{\prime}$. Now, let $x$ be a material object in space; then $\operatorname{pro}(x, n, \lambda y \bigvee l(y$ is at $l), t)=1$, that is, the sum of $\operatorname{pro}(x, n$, being-at $-l, t)$ over all $l$ is 1 ; and apparently there is a set of non-denumerably many locations, such that for all members $l, l^{\prime}$ of that set $\operatorname{pro}(x, n$, being-at- $l, t)>0$ and $\operatorname{pro}(x, n$, being-at- $l, t)=\operatorname{pro}\left(x, n\right.$, being-at- $\left.l^{\prime}, t\right)$. But clearly this cannot be, and the fact that it cannot be is not contra-intuitive. Indeed, if it is guaranteed at $n$ that there is no force acting on $x$ between $n$ and $t$ Newton's Law tells us: $\bigvee l(p r o(x, n$, being-at- $l, t)=$ $1 \wedge \wedge l^{\prime}\left(l^{\prime} \neq l \supset \operatorname{pro}\left(x, n\right.\right.$, being-at- $\left.\left.\left.l^{\prime}, t\right)=0\right)\right)$. (There is no such nice solution to the problem if you consider it mutatis mutandis for subjective probabilities. Why shouldn't I now think it equally possible for non-denumerably many locations that $x$ is at them at $t$ ? But no, I can't rationally do this. This seems strange.)

\section{REFERENCES}

Armstrong, D. M.: 1978, Universals and Scientific Realism, 2 vol., Cambridge University Press, Cambridge, England. 
Bealer, G.: 1982, Quality and Concept, Clarendon Press, Oxford, England.

Galavotti, M. C.: 1987, 'Comments on Patrick Suppes "Propensity Interpretation of Probability"', Erkenntnis 26, 359-368.

Giere, R. N.: 1979, 'Propensity and Necessity', Synthese 40, 439-451.

Hacking, I.: 1971, 'The Leibniz-Carnap Program for Inductive Logic', Journal of Philosophy 68, 597-610.

Humphreys, P.: 1985, 'Why Propensities cannot be Probabilities', The Philosphical Review 94, 557-570.

Kenny, A.: 1980, Aquinas, Oxford University Press, Oxford, England.

Lewis, D.: 1986, On the Plurality of Worlds, Blackwell, Oxford, England.

Smokler, H.: 1979, 'Single-case Propensities, Modality and Confirmation', Synthese 40, 497-506.

Manuscript submitted February 12, 1992

Final version received September 28, 1992

Universität Regensburg

Philosophisches Seminar

Universitätsstr. 31

8400 Regensburg

Germany 